\title{
DigApp and TaphonomApp: Two new open-access palaeontological and archaeological mobile apps
}

\author{
David M. Martín-Perea, Ana Abrunhosa, M. Soledad Domingo, Enrique Cantero, \\ Iris Menéndez, Fernando Blanco, Patricia M. Carro-Rodríguez, Laura Domingo, \\ Manuel Hernández Fernández, and Jorge Morales
}

\begin{abstract}
Two new paleontological and archaeological Android applications, DigApp and TaphonomApp, are presented in this manuscript. DigApp is intended to aid data collection, storage and management in archaeological and palaeontological excavations. DigApp allows easily recording of common field information such as spatial data and fossil identification data. Online and offline versions of DigApp were developed to fit all needs, and they can be modified according to the excavation particularities. TaphonomApp was created in order to assist taphonomists while carrying out detailed taphonomical evaluations both in the field and in the laboratory, making data collection quicker, homogeneous and overall, more efficient. DigApp and TaphonomApp are free, open-access and flexible software, that can be easily modified by any user (without the need of expertise in computing or coding) as explained in this paper. An in-depth guide on how to modify the apps is provided within this paper. DigApp and TaphonomApp have been used during palaeontological excavations carried out at one of the Batallones Butte vertebrate sites (Batallones-10, Middle Miocene) in the Madrid basin (Spain).
\end{abstract}

David M. Martín-Perea. Palaeobiology Department, National Museum of Natural Sciences - CSIC. Madrid, 28006, Spain; Geodynamics, Stratigraphy and Palaeontology Department, Faculty of Geological Sciences, Complutense University of Madrid, Madrid, 28040, Spain; IDEA - Institute of Evolution in Africa, University of Alcalá de Henares, Madrid, 28010, Spain. davidmam@ucm.es

Ana Abrunhosa. Interdisciplinary Centre for Archaeology and Evolution of Human Behaviour, Faculty of Human and Social Science, Algarve University, Faro, 8005-139, Portugal; Archaeological Regional Museum, Alcalá de Henares, 28801, Spain. ana.abrunhosa@gmail.com

M. Soledad Domingo. Geodynamics, Stratigraphy and Palaeontology Department, Faculty of Geological Sciences, Complutense University of Madrid, Madrid, 28040, Spain; Experimental Sciences, Social

Martín-Perea, David M., Abrunhosa, Ana, Domingo, M. Soledad, Cantero, Enrique, Menéndez, Iris, Blanco, Fernando, CarroRodríguez, Patricia M., Domingo, Laura, Hernández Fernández, Manuel, and Morales, Jorge. 2020. DigApp and TaphonomApp: Two new open-access palaeontological and archaeological mobile apps. Palaeontologia Electronica, 23(2):a28. https://doi.org/10.26879/ 1043

palaeo-electronica.org/content/2020/3085-digapp-and-taphonomapp

Copyright: June 2020 Paleontological Society.

This is an open access article distributed under the terms of Attribution-NonCommercial-ShareAlike 4.0 International (CC BY-NC-SA 4.0), which permits users to copy and redistribute the material in any medium or format, provided it is not used for commercial purposes and the original author and source are credited, with indications if any changes are made.

creativecommons.org/licenses/by-nc-sa/4.0/ 
Sciences and Mathematics Department, Faculty of Education, Complutense University of Madrid, Madrid, 28040, Spain.soldomingo@gmail.com

Enrique Cantero. Palaeobiology Department, National Museum of Natural Sciences - CSIC. Madrid, 28006, Spain. canteroycantero@hotmail.com

Iris Menéndez. Geodynamics, Stratigraphy and Palaeontology Department, Faculty of Geological

Sciences, Complutense University of Madrid, Madrid, 28040, Spain; Sedimentary Geology and

Environmental Change Department, Geosciences Institute - CSIC, Complutense University of Madrid,

Madrid, 28040, Spain. irismene@ucm.es

Fernando Blanco. Natural History Museum, Berlin, 10115, Germany. fblancosegovia@gmail.com

Patricia M. Carro-Rodríguez. Geodynamics, Stratigraphy and Palaeontology Department, Faculty of

Geological Sciences, Complutense University of Madrid, Madrid, 28040, Spain; Sedimentary Geology and

Environmental Change Department, Geosciences Institute - CSIC, Complutense University of Madrid,

Madrid, 28040, Spain. patcarro@ucm.es

Laura Domingo. Geodynamics, Stratigraphy and Palaeontology Department, Faculty of Geological

Sciences, Complutense University of Madrid, Madrid, 28040, Spain; Sedimentary Geology and

Environmental Change Department, Geosciences Institute - CSIC, Complutense University of Madrid, Madrid, 28040, Spain; Earth and Planetary Sciences Department, University of California Santa Cruz, Santa Cruz, California, 95064, USA. lauradomingo@geo.ucm.es

Manuel Hernández Fernández. Geodynamics, Stratigraphy and Palaeontology Department, Faculty of Geological Sciences, Complutense University of Madrid, Madrid, 28040, Spain; Sedimentary Geology and Environmental Change Department, Geosciences Institute - CSIC, Complutense University of Madrid, Madrid, 28040, Spain. hdezfdez@ucm.es

Jorge Morales. Palaeobiology Department, National Museum of Natural Sciences - CSIC. Madrid, 28006, Spain.mcnm166@mncn.csic.es

Keywords: Taphonomical analysis; archaeological site; palaeontological site; MIT App Inventor; Android; smartphone app

Submission: 14 November 2019. Acceptance: 21 June 2020.

\section{INTRODUCTION}

Smartphones and tablets have become essential in our daily lives. We constantly use these devices to communicate, organize ourselves, annotate ideas, know our exact location or take photographs. We have replaced traditional tools such as agendas, notebooks, GPS devices and cameras with their mobile application counterparts developed for these multi-role, pocket-sized gadgets. This paradigm change has also extensively happened in science (McPherron et al., 2003; Newman et al., 2012; Young, 2012). Geologists have replaced field notebooks, GPS or compasses with mobile tools, which allow them to acquire data more efficiently. First, the data are homogeneous, even if they are acquired by different researchers. This removes substantially the human error and makes the process of data gathering faster, as the step of transcribing the handwritten data of the fieldwork to digital data is removed (Weng et al., 2012). Second, the cost of the fieldwork is reduced, as many mobile apps are free and open-access. Lastly, as different functions can be combined in one device, it is not necessary to carry a large number of tools to the fieldwork (Sun et al., 2010; Weng et al., 2012).

In any event, the use of open and free mobile apps in archaeology and palaeontology is not a widespread practice yet. Data recording methods in palaeontological and archaeological excavations have slowly evolved, and traditional working protocols are still being used in the vast majority of excavations. This is surprising since data recovered during the excavation and extraction of fossil and lithic remains are of vital importance (Lyman, 1994; Eberth et al., 2007) and, given the high number of fossils recovered at some sites, researchers would greatly benefit from the use of these apps. Data recording, along with a clear strategy and organization of the excavation should be considered of upmost importance and the use of mobile apps could greatly optimize this task (Morgan and Eve, 2012; Reed et al., 2015).

Mainly over the last three decades, researchers working in different archaeological and palaeontological projects have developed methods to 
digitally record fossiliferous data while excavating. For example, Canals et al. (2008) proposed a technological solution to overcome the aforementioned shortcomings of traditional recording protocols, applied at the archaeopalaeontological excavations taking place in the Sierra de Atapuerca sites. The 3COORsystem (Canals et al., 2008) includes a series of subsystems and software, and makes use of Personal Digital Agendas (PDAs) for data input, a laptop as a server and central data repository and Bluetooth and $\mathrm{Wi}-\mathrm{Fi}$ wireless technologies to connect all of these devices.

However, this technological solution is neither free (which restrains its use to the availability of funding sources) nor open-access (hindering the evolution, development and customization of the software) and, therefore, it is exclusively used in the Sierra de Atapuerca sites. Besides the hardware and software costs associated (Sosna et al., 2014) in many cases, these methodologies and their outcomes can only be handled (and understood) by professionals with an ample knowledge of specialized software and computing techniques.

We present here two completely free, openaccess and easy-to-use mobile apps to assist archaeologists, palaeontologists and geologists in the field and during data collection: DigApp and TaphonomApp. These apps are available for download on the website (https://dmartinperea.com/ digapp-taphonomapp/).

Traditionally, the creation of such mobile apps was complex, requiring proficiency on the use of software programming codes and specialized development environments. This restricted the possibility to create apps to just a minority of the population. However, the creation of the App Inventor by Google, later revisited, rescued and improved by the Massachusetts Institute of Technology (MIT, Cambridge, USA) resulted in a technological revolution, allowing everyone to easily develop their own personalized apps.

MIT App Inventor is a simple way of creating mobile applications for Android systems. It is a freeware application run directly from a web browser, which uses intuitive "building blocks" instead of complicated traditional software programming codes. Although MIT App Inventor is currently offered in a Beta version, it is fully operational and can be found at http://ai2.appinventor.mit.edu/.

As predicted by Cascalheira et al. (2014), MIT App Inventor has become a very useful tool for archaeological and palaeontological projects. This is best exemplified by ArchaeoSurvey, Archaeo-
Survey Raw Materials Edition and Lithics On The Go, three apps developed by Cascalheira et al. (2014, 2017) and Abrunhosa et al. (2017), which were designed to support archaeological field surveys and studies, combining advanced computing capabilities and connectivity of smartphones with customized (and further customizable) mobile apps. These apps were the inspiration for the creation of the apps presented in this paper.

Other projects have also made good use of mobile application software with the purpose of collecting field data and dealing with bid databases. Some examples are those of the Geological Survey of Denmark and Greenland for the description of geological localities (Hansen et al., 2016), the paperless recording methodology at the Sangro Valley Project (Motz and Carrier, 2013), the use of bar codes associated with three-dimensional coordinates taken with a computer-linked total station on-site (Dibble et al., 2007), the Proyecto de Investigación Arqueológico Regional Ancash (PIARA) in Peru (DeTore and Bria, 2012) and the geochemical rock-sourcing projects in the USA (Goodale et al., 2013).

\section{DIGAPP}

DigApp is a mobile application set up to facilitate data collection at palaeontological and archaeological excavations. The app has been designed to be as accessible, intuitive and easy to use as possible and can be downloaded from the website https://dmartinperea.com/digapp-taphonomapp/.

DigApp is run using a Graphical User Interface (GUI) and is divided into three different screens: "Home", "New Specimen" and "View Specimens" (Figure 1).

\section{DigApp's Interface}

As soon as DigApp is initialized, the "Home" screen (Figure 1A) appears. This simple screen allows the user to advance on to the "New Specimen" screen, to the "View Specimen" screen or to exit the app via the "Back" button.

The "New Specimen" screen (Figure 1B) is the backbone of the app. From this screen, all data is recorded, either by filling in blank spaces using text (such as "Specimen Number", " $X$ ", "Y" and "Z" values) or by selecting available options from a dropdown list (such as "Anatomical Identification" or "Taxonomical Identification"). DigApp has 11 predetermined fields for data input: specimen number, anatomical identification, taxonomical identification, grid (separated into two dropdown menus for easier data input), $x$ value, $y$ value, $z$ value, 

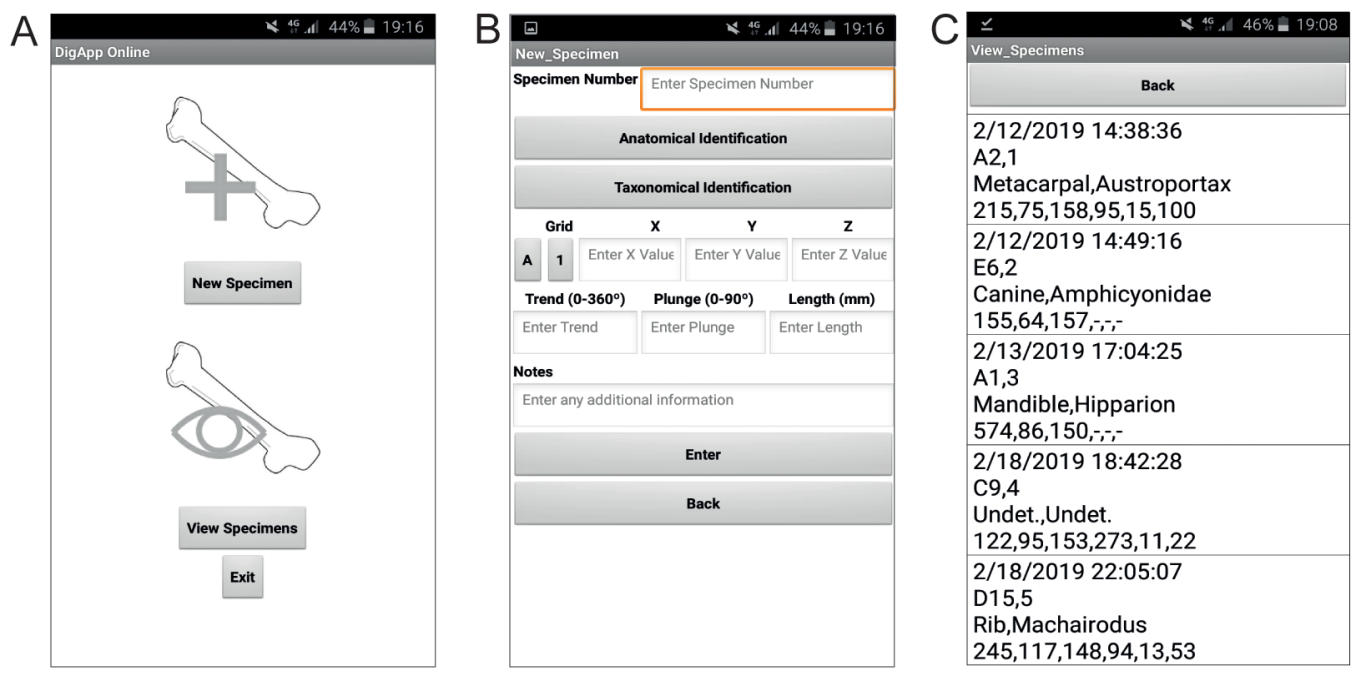

FIGURE 1. A, DigApp Online "Home" screen. B, DigApp Online "New Specimen" screen. C, DigApp Online "View Specimens" screen.

trend, plunge, length and notes. These fields were chosen according to standard techniques commonly used in vertebrate fossil excavations (Lyman, 1994; Eberth et al., 2007).

Once the fields are filled with the desired information, and the "Enter" button is pressed, the data will automatically be saved in the cloud in a Google Spreadsheet. In order to avoid any erroneous data inputs, the app is programmed to show a warning message when "Specimen Number", "Anatomical Identification", "Taxonomical Identification", " $X$ ", " $Y$ " or " $Z$ " fields are either left unfilled or incorrectly filled (for example, entering a non-numerical value for the spatial information such as " $X$ ", "Y", " $Z$ " or the trend, plunge and length), since these fields are deemed too important for the integrity of the database to be left blank or incorrectly filled.

Finally, the "View Specimens" screen (Figure 1C) has been created for DigApp's online version to keep track of the data that have been already successfully recorded. The list is updated when the "View table" button is pressed, showing every entry previously recorded in the Google Spreadsheets table.

\section{Modifying DigApp}

DigApp is a free, open-access mobile application. As such, the .aia file (a source code file that can be opened and created with MIT App Inventor) is made available to be freely modified on MIT App Inventor or any other mobile application creation software. Modifications can range from adjusting the faunal list displayed in the "Taxonomical Identification" dropdown menu, to adding a new variable.
An in-depth guide can be followed in order to customize DigApp in MIT App Inventor to best fit the users' needs and to set the URL for the Google Spreadsheet (Supplementary Information 1). Furthermore, two modified versions of DigApp (DigApp Offline and DigApp Easy To Use) are available.

DigApp "Offline". This modified version of the app is exactly the same as DigApp Online, with the exception that the data is saved in the mobile device's memory storage as a comma-separated values table (.csv file). This app has been developed to be used in sites where an internet connection is not available.

DigApp Easy To Use. An alternate version of DigApp has been created for users who do not have the time or ability to modify the app. This version is ready to be used as soon as it is downloaded, without the need of setting the Google Spreadsheet's URL as in DigApp Online or adjusting the dropdown menus, as in DigApp Online and DigApp Offline. For this reason, dropdown menus, which need to be adjusted to the site's faunal assemblage, have been replaced with empty text boxes, to be filled in freely by the user, and the data is saved in the mobile device's memory storage as a comma-separated values table (.csv file) as in DigApp Offline.

\section{Using DigApp at Batallones-10 Palaeontological Site}

DigApp was initially tested during the excavation of Batallones-10 vertebrate site (Madrid basin, Spain) in the field season of 2018. Since internet 

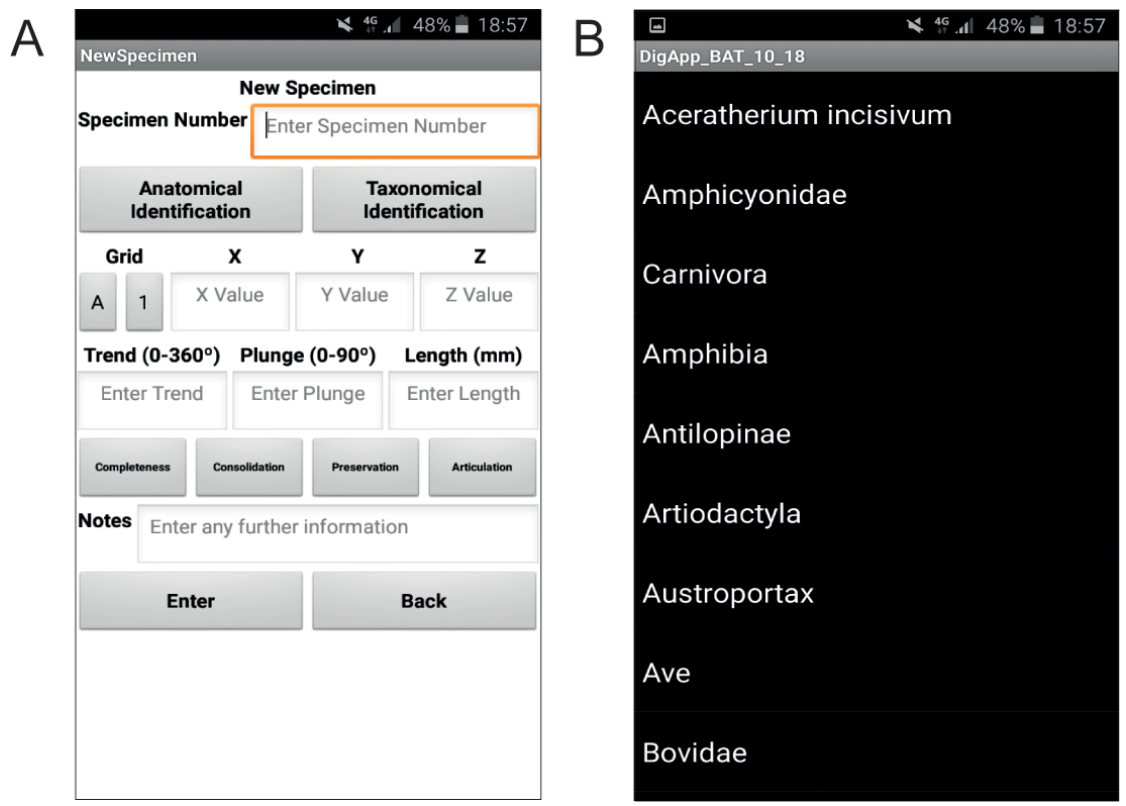

FIGURE 2. DiggApp Offline modified for excavations at Batallones-10 palaeontological site. A "New Specimen" screen, with "Completeness", "Consolidation", "Preservation" and "Articulation" fields added. B, "Taxonomical Identification" dropdown menu modified to include Batallones-10 faunal list.

connectivity was very deficient in the area, a modified version of DigApp Offline was used (Figure 2), with three new variables traditionally registered at the site ("Completeness", "Consolidation" and "Preservation") included (Figure 2A) and the "Taxonomical Identification" dropdown menu adjusted to include all taxa in the assemblage (Figure 2B).

A single tablet device, with the application installed, was used to collect excavation data throughout the site. At the same time, data was recorded on paper sheets as a backup, in case any malfunction would occur.

\section{TAPHONOMAPP}

As already mentioned, the fact that DigApp is open-access, grants the user the ability to modify the software. These modifications, interestingly, are not limited to only creating variants of DigApp to use at archaeological and palaeontological excavations: the software can be modified to suit data collection of any set of data from any field. An example of this is the creation of TaphonomApp, a mobile application, which assists taphonomists while carrying out taphonomical analyses, normally consisting of a large quantity of variables.

\section{TaphonomApp's Interface}

TaphonomApp's interface is very similar to that of DigApp, following the same principle of being simplistic, easy to use and extremely intui- tive. It is divided into three different screens: "Home", "Taphonomical Analysis" and "New Specimen" (Figure 3).

When TaphonomApp is initialized, the "Home" screen appears (Figure 3A). This simple screen, very similar to DigApp's "Home" screen, allows the user to advance on to the "Taphonomical Analysis" screen or to exit the app by pressing the "Back" button. The "Home" screen, which apparently seems to serve no purpose, has been created in order to introduce in a future version of the app a "Taphonomical Transect" feature, a taphonomical variant of Cascalheira et al. $(2014,2017)$ and Abrunhosa et al. (2017) app ArcheoSurvey. Taphonomical transects are carried out in order to monitor animal carcasses and bones in modern ecosystems and compare faunal fidelity of the bone assemblage to the living community, which allows to document the processes governing death, decomposition and potential preservation or destruction of the carcasses (Weigelt, 1927; Behrensmeyer and Hill, 1980; Behrensmeyer and Kidwell, 1985; Kidwell and Flesa, 1995; Behrensmeyer et al., 2000; Western and Behrensmeyer, 2009; Miller, 2011; Behrensmeyer and Miller, 2012; Kidwell and Tomasovych, 2013; Domingo et al., 2018).

The "Taphonomical Analysis" screen (Figure $3 A$ ) allows the user to advance on to the "New Specimen" screen or back to the "Home" screen. 

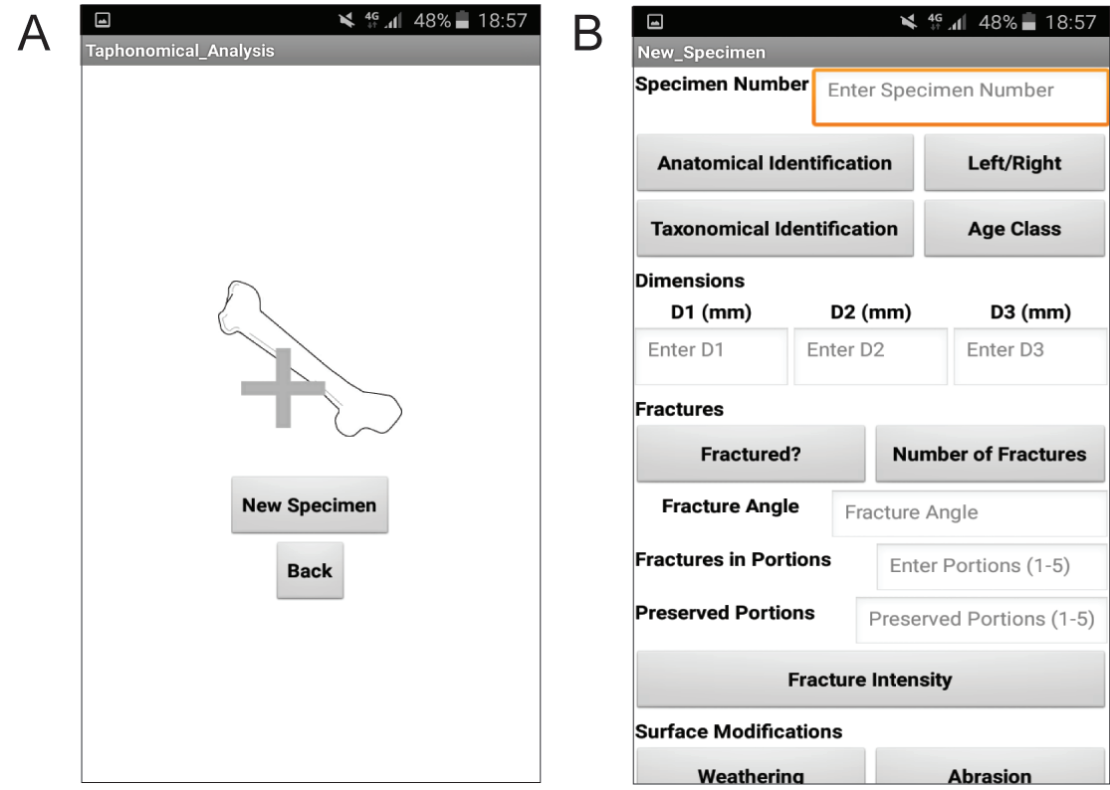

FIGURE 3. A, TaphonomApp "Taphonomical Analysis" screen. B, TaphonomApp "New Specimen” scrollable screen.

From the "New Specimen" screen (Figure 3B), the user enters all data for the analysed specimen. Data is recorded either by filling in blank spaces using text (such as for "Specimen Number" or "Notes") or by selecting available options from a dropdown list (such as for "Anatomical Identification" or "Taxonomical Identification"). TaphonomApp has 31 predetermined fields for data input, which are discussed below.

Specimen number. To enter the Specimen number, the user has to type in a blank space the desired value. Data entry can be modified in MIT App Inventor to add a prefix (such as "BAT10-" for "Batallones-10") to the data entered in the Google Spreadsheet or .csv table.

Anatomical identification. The user selects the anatomical part from a dropdown menu, which displays an anatomical list. This anatomical list can be easily modified in MIT App Inventor according to the user's needs.

Left/right. A simple dropdown menu is used to determine whether limb or mandibular elements are left or right-sided. For axial bones (e.g., vertebrates), this field is left blank.

Taxonomical identification. The user selects the taxonomical identification of the studied specimen from a dropdown menu that displays a taxonomical list, which can be easily modified in MIT App Inventor to include taxa from the assemblage being analysed.

Age class. A dropdown menu will allow the user to select from several ontogenetic stages (Infant,
Juvenile, Adult and Senile). This field can be left blank if ontogenetic age is undetermined.

D1 length, D2 length, D3 length. These perpendicular measurements (in millimetres) defined by Blott and Pye (2008) in order to describe morphometrically the specimen as a sedimentary particle, are entered in a blank space.

Fracture variables. Several taphonomical variables related to fracturing are addressed in this section, following Villa and Mahieu (1991) and Lyman (1994). Clicking on "Fractured" will display a dropdown menu with "yes" or "no" options. If the bone is fractured, the next field, "Number of fractures" displays another dropdown menu to select the number of fractures $(1,2,3,4,>5)$. A blank field is left to input "Fracture angle", which refers to perpendicular fracture $(A)$, oblique fracture $(B)$ and parallel fracture (C). An additional blank field, "Fracture location", is used to describe, for long bones, where the fractures are located, whether in the proximal epiphysis (1), proximal end of the diaphysis (2), medial part of the diaphysis (3), distal end of the diaphysis (4) or distal epiphysis (5). In the "Preserved Portion" field, the user inputs what part of the bone is preserved, following the same procedure as for "Fracture location" (i.e., "15 " would describe a complete bone, whereas "2-5" would describe an incomplete bone missing the proximal diaphysis). "Fracture intensity" refers to complete (Stage 0), almost complete (Stage 1, only missing some bone chips), more than one-half 
complete (Stage 2) and one-half or less complete (Stage 3) bones.

Weathering. A dropdown menu displays the scale proposed by Behrensmeyer (1978) to evaluate bone weathering. Each stage on the scale can be related to the number of years from death to burial: Stage 0 (0-1 year), Stage 1 (6 months -2.5 years), Stage 2 (2-4 years), Stage 3 (4-8 years), Stage 4 (6.5-20 years) and Stage 5 ( $10->25$ years).

Abrasion. Abrasion refers to the smoothing and polishing of bones, which can be classified into Stage 0 (intact bone), Stage 1 (moderately rounded bone) and Stage 2 (extremely polished bone) as proposed by Alcalá (1994).

Other taphonomical variables. Simple dropdown menus, displaying "Yes" and "No" have been introduced for taphonomical variables such as corrosion, digestion, trampling, manganese oxide presence, root marks and rodent marks.

Carnivore marks. "Carnivore Marks" displays a dropdown menu with "Yes" and "No" options, to indicate presence or absence of carnivore marks. A blank field is allocated for "Carnivore Mark Type", where nomenclature proposed by Blumenschine (1995) should be followed. Using the same procedure as for "Fracture location", "Carnivore Mark in Portion" is used to describe the location of the mark.

Cut marks. "Cut Marks" displays a dropdown menu with "Yes" and "No" options, to indicate presence or absence of cut marks according to criteria defined by (Domínguez-Rodrigo et al., 2009). Using the same procedure as for "Fracture location", "Cut Marks in Portion" is used to describe the location of the mark.

Percussion marks. "Percussion Marks" displays a dropdown menu with "Yes" and "No" options, to indicate presence or absence of percussion marks, following Blumenschine and Selvaggio (1988) and Blumenschine (1995). Using the same procedure as for "Fracture location", "Cut Marks in Portion" is used to describe the location of the mark.

Photograph. "Photographed" displays a simple "Yes" and "No" dropdown menu to state whether or not the studied specimen has been photographed. Photograph details can be recorded in the "Notes" field below.

Notes. A blank field at the end of the form is intended for any additional information the user might want to add.

After filling desired fields with the taphonomical information and pressing the "Enter" button, the data will automatically be saved in a .csv table in the device's internal storage. As with all versions of
DigApp, the app is programmed to show a warning message when the "Specimen Number", "Anatomical Identification" or "Taxonomical Identification" fields are left unfilled.

\section{DISCUSSION}

DigApp and TaphonomApp's main advantages are efficiency related. These mobile applications simplify and unify different tasks within one user-friendly software in a single electronic device. These apps bring forward data digitalization into the field, and make the data readily available, granting the ability to obtain real-time palaeontological, archaeostratigraphic, geoarchaeological and spatial analytic information to support and enhance fieldwork.

The use of DigApp and TaphonomApp is a paperless system, which replaces more traditional field data recording using paper forms. Traditionally, paper forms were subsequently transcribed into computer databases, a very time-consuming task. The use of the apps is not only eco-friendly, since it avoids the use of paper, but also reduces considerably the time spent in such repetitive tasks.

Errors introduced in data transcription and recording of the traditional paper forms are very common, such as errors derived from interpreting handwriting. These errors are significantly reduced with the use of these apps, decreasing humandriven errors through automation. The use of dropdown menus, instead of manually entering text in blank fields avoid inputting erroneous text and improves the consistency of terminology, creating a homogeneous database.

DigApp Online version has been linked to a Google Spreadsheet, making the data easily and readily available anywhere once it is collected from the field. This not only improves the overall efficiency of data recording at the archaeological or palaeontological excavation, but it also gives rise to an improvement of the security of the data collected, since synchronization is continuous. Furthermore, this online version of the application can be used to work simultaneously with several devices in any number of sites.

On the other hand, it is strongly recommended to manually create a daily back-up of the data stored in the device's storage through the use of offline versions such as DigApp Offline, DigApp Easy to Use and TaphonomApp in order to avoid any, although unlikely, data loss.

DigApp has several advantages over other software, such as the aforementioned advanced 
3COORsystem used at the Atapuerca sites. First and foremost, DigApp is completely free and customizable, allowing the user to modify and cater the app to her or his needs. The apps are run in any android mobile device without the need of a laptop. Furthermore, the apps consume less energy since there is no need to connect to a central data repository via Wi-Fi or Bluetooth.

The use of DigApp at Batallones-10 turned out to be a more efficient way of data recording. Once the excavation was over, time traditionally spent in data transcription was instead invested in data analysis. Moreover, the database created was homogeneous, making data manipulation easier and more effective.

Finally, DigApp and TaphonomApp are in constant development and will continually evolve to comply with new requirements. For example, the inclusion of photographs or sketch drawings for some entries could be very helpful and would further improve efficiency both at the field and in posterior data analyses. The implementation of these apps for iOS devices is currently under way. Since the applications are open software, completely modifiable and easy to use, it is our hope that a user-driven community will emerge and develop largely both apps, eventually leading to the creation of other new, creative and scientifically useful apps in the archaeological and palaeontological fields.

While the value of this contribution is that many specialists willing to use the app do not have to have prior programming experience, those users who do could take these apps a step further, developing certain aspects of the system. MIT App Inventor is written in Java, Kawa and Scheme, allowing programmers to develop the app beyond what is made readily available by MIT App Inventor.

\section{ACKNOWLEDGEMENTS}

We would like to thank all Batallones-10 field season participants who have helped test the initial versions of these apps. This research has been funded by Project CGL2015-68333-P of the Spanish Government. DMMP was funded by a FPI Predoctoral grant BES-2016-079460 from the Spanish Government associated to Project CGL201568333-P. AA was funded bu a doctoral grant (SFRH/BD/110511/2015) from Fundação para a Ciência e Tecnologia (FCT - Portuguese Science Foundation) and a Dissertation Fieldwork Grant funded by Wenner-Gren Foundation for the Project "Raw Material Procurement Strategies from Pinilla del Valle's Neanderthals". IM was funded by a Predoctoral grant from the Complutense University of Madrid (CT27/16-CT28/16). FB was funded by a research grant from Deutsche Forschungsgemeinschaft (LO 2368/1-1). PMCR was funded by a Predoctoral grant from the Complutense University of Madrid (CT42/18-CT43/18). This is partly a contribution of the research group UCM910607.

\section{REFERENCES}

Abrunhosa, A., Cascalheira, J., Pérez-González, A., Arsuaga, J.L., and Baquedano, E. 2017. The use of digital mobile technologies for Geoarchaeological survey: the example of the Pinilla del Valle raw materials project. 12th International Conference of Archaeological Prospection, Bradford, p. 1-4.

Alcalá, L. 1994. Macromamíferos Neógenos de la Fosa de Alfambra-Teruel. Unpublished PhD Thesis, National Natural Sciences Museum, Madrid, Spain.

Behrensmeyer, A.K. 1978. Taphonomic and ecologic information from bone weathering. Paleobiology, 4:150-162. https://doi.org/10.1017/s0094837300005820

Behrensmeyer, A.K. and Hill, A.P. (eds.). 1988. Fossils in the Making: Vertebrate Taphonomy and Paleoecology 69. University of Chicago Press, Chicago.

Behrensmeyer, A.K. and Kidwell, S.M. 1985. Taphonomy's contributions to paleobiology. Paleobiology, 11(1):105-119. https://doi.org/10.1017/s009483730001143x

Behrensmeyer, A.K, Kidwell, S.M., and Gastaldo, R.A. 2000. Taphonomy and paleobiology. Paleobiology, 26:103-147. https://doi.org/10.1017/s0094837300026907

Behrensmeyer, A.K. and Miller, J.H. 2012. Building links between ecology and paleontology using taphonomic studies of recent vertebrate communities, p. 69-91. In Louys, J. (ed.), Paleontology in Ecology and Conservation. Springer-Verlag, Berlin and Heidelberg. https:// doi.org/10.1007/978-3-642-25038-5_5 
Blott, R.W. and Pye, K. 2008. Particle shape: a review and new methods of characterization and classification. Sedimentology, 55:31-63. https://doi.org/10.1111/j.1365-3091.2007.00892.x

Blumenschine, R.J. 1995. Percussion marks, tooth marks and the experimental determination of the timing of hominid and carnivore access to long bones at FLK Zinjanthropus, Olduvai Gorge, Tanzania. Journal of Human Evolution, 29:21-51. https://doi.org/10.1006/ jhev.1995.1046

Blumenschine, R.J. and Selvaggio, M.M. 1988. Percussion marks on bone surfaces as a new diagnostic of hominid behavior. Nature, 333:763-765. https://doi.org/10.1038/333763a0

Canals, A., Rodríguez, J., and Sánchez, R. 2008. The 3COORsystem for data recording in archaeology. Journal of Anthropological Sciences, 86:133-141.

Cascalheira, J., Gonçalves, C., and Bicho, N. 2014. Smartphones and the use of customized apps in archaeological projects. The SAA Archaeological Record, 13(5):20-25.

Cascalheira, J., Gonçalves, C., and Bicho, N. 2017. A Google-based freeware solution for archaeological field survey and on-site artifact analysis. Advances in Archaeological Practice, 5(4):328-339. https://doi.org/10.1017/aap.2017.21

DeTore, K. and Bria, R. 2012. Digital Archaeology at PIARA. Electronic document, accessed 22 November 2019. PIARA. http://www.piaraperu.org

Dibble, H.L., Marean, C.W., and McPherron, S.P. 2007. The use of barcodes in excavation projects. The SAA Archaeological Record, 7:33-38.

Domingo, M.S., Martín-Perea, D.M., Badgley, C., and Negro, J.J. 2018. The bones of Doñana National Park (Spain): post-mortem modifications in bones and carcasses from a modern Mediterranean ecosystem. 5th International Palaeontological Congress, Paris, p. 979.

Domínguez-Rodrigo, M., Juana, S., Galán, A.B., and Rodríguez, M. 2009. A new protocol to differentiate trampling marks from butchery cut marks. Journal of Archaeological Science, 36(12):2643-2654. https://doi.org/10.1016/j.jas.2009.07.017

Eberth, D.A., Rogers, R.R., and Fiorillo, A.R. 2007. A practical approach to the study of bonebeds, p. 265-331. In Rogers, R.R., Eberth, D.A., and Fiorillo, A.R. (eds.), Bonebeds; Genesis, Analysis and Paleobiological Significance. University of Chicago Press, Chicago.

Goodale, N., Bailey, D., Fondak, T., and Nauman, A. 2013. iTROWEL. Mobile devices and transformative technology in archaeological field research. The SAA Archaeological Record, 13(3):18-22.

Hansen, M., Petersen, M.N., Kokfelt, T.F., and Stensgaard, B.M. 2016. aFieldWork - an Android app for offline recording of geological information and data display. Geological Survey of Denmark and Greenland Bulletin, 35:99-102. https://doi.org/10.34194/geusb.v35.4949

Kidwell, S.M. and Flessa, K.W. 1995. The quality of the fossil record: populations, species, and communities. Annual Review of Ecology, Evolution and Systematics, 26:269-299. https:// doi.org/10.1146/annurev.earth.24.1.433

Kidwell, S.M. and Tomasovych, A. 2013. Implications of time-averaged death assemblages for ecology and conservation biology. Annual Review of Ecology, Evolution and Systematics, 44:539-563. https://doi.org/10.1146/annurev-ecolsys-110512-135838

Lyman, R.L. 1994. Vertebrate Taphonomy. Cambridge University Press, Cambridge. https:// doi.org/10.1017/cbo9781139878302

McPherron, P.S. and Diblle, H.L. 2003. Using computers in adverse field conditions: Tales from the Egyptian Desert. The SAA Archaeological Record, 3(5):28-33.

Miller, J.H. 2011. Ghosts of Yellowstone: multi-decadal histories of wildlife populations captured by bones on a modern landscape. PLoS ONE, 6(3):e18057. https://doi.org/10.1371/ journal.pone.0018057

Morgan, C. and Eve, S. 2012. DIY and digital archaeology: what are you doing to participate? World Archaeology, 44:521-537. https://doi.org/10.1080/00438243.2012.741810

Motz, C.F. and Carrier, S.C. 2013. Paperless recording at the Sangro Valley Project, p. 25-30. In Earl, G., Sly, T., Chrysanthi, A., Murrieta-Flores, P., Papadopoulos, C., Romanowska, I., and Wheatley, D. (eds.), Archaeology in the Digital Era - 40th Annual Conference of Computer Applications and Quantitative Methods in Archaeology (CAA). Amsterdam University Press, Amsterdam. https://doi.org/10.1515/9789048519590-003

Newman, G., Wiggins, A., Crall, A., Graham, E., Newman, S., and Crowston, K. 2012. The future of citizen science: emerging technologies and shifting paradigms. Frontiers in Ecology and the Environment, 10(6):298-304. https://doi.org/10.1890/110294 
Reed, D., Barr, W.A., McPherron, S.P., Bobe, R., Geraads, D., Wynn, J.G., and Alemseged, Z. 2015. Digital data collection in paleoanthropology. Evolutionary Anthropology: Issues, News, and Reviews, 24(6):238-249. https://doi.org/10.1002/evan.21466

Sosna, D., Brunclíková, L., and Henig, D. 2013. Testing iPad in the field: use of a relational database in garbological research. Anthropologie, 51(3):421-430.

Sun, F.S., Weng, Y.H., and Grigsby, J.D. 2010. Smartphones for geological data collection - an Android phone application. Eos, 91:59.

Villa, P. and Mahieu, E. 1991. Breakage patterns of human long bones. Journal of Human Evolution, 21:27-48. https://doi.org/10.1016/0047-2484(91)90034-S

Weng, Y.H., Sun, F.S., and Grigsby, J.D. 2012. GeoTools: An android phone application in geology. Computers and Geosciences, 44:24-30. https://doi.org/10.1016/ j.cageo.2012.02.027

Weigelt, J. 1927. Recent Vertebrate Carcasses and Their Paleobiological Implications. University of Chicago Press, Chicago. (Translated by Schaefer, J. 1989)

Western, D. and Behrensmeyer, A.K. 2009. Bone assemblages track animal community structure over 40 years in an African savanna ecosystem. Science, 324:1061-1064. https:// doi.org/10.1126/science. 1171155

Young, H.A. 2012. Scientific Apps are here (and more will be coming). Cytokine, 59(1):1-2. https://doi.org/10.1016/j.cyto.2012.02.014 